\title{
Schutz vor pathogenen Viren?
}

\author{
Nicht nur bei Durchfall.
}

Die Zahl der humanpathogenen Erreger ist noch nicht genau bekannt. Die Fachzeitschrift "Nature" postuliert an die 1400 Pathogene, davon 208 Viren (Beispiele in - Abb. 1) und Prionen, 538 Bakterien, 317 Pilzarten, 57 Protozoen und 287 Wurmarten. Andere Schätzungen postulieren eine sehr viel größere Zahl gefährlicher Mikroben es könnten über 100.000 oder noch viel mehr verschiedene Angreifer sein, die von der Forschung erst dechiffriert werden müssen.

Den besten Schutz gegen gefährliche Viren bieten Impfstoffe, die die Erkrankungen gar nicht ausbrechen lassen. Die bisherige Palette der Vakzine schützt uns aber lediglich vor rund 70 Pathogenen. Alle anderen muss unsere unspezifische Immunabwehr aufhalten oder völlig inaktivieren.

Die mütterlichen Laktobazillen bilden nach der Geburt einen Rucksack für mehrere Jahre, der die Immunabwehr entscheidend trainiert. Die Mikrobiologie kennt zwischenzeitlich über 300 verschiedene Milchsäurebakterien, von denen allerdings nur wenige therapeutisch genutzt werden können. In Österreich gibt es gerade sechs Probiotika mit ArzneimittelZulassungen.

\section{Das Breitband-Probiotikum}

Einige Keime haben eine besonders starke Wirkung auf das Immunsystem. Diese Laktobazillen bestechen durch klinische Effekte gegen eine ganze Zahl von Bakterien und Viren gleichzeitig. Wissenschaftlich gut dokumentiert sind die Milchsäurebakterien L. casei rhamnosus (LCR35).

Die finnische Kinderärztin Prof. Erika Isolauri und die französische Mikrobiologin Prof. Christiane Forestier postulieren, dass die Bakteriengruppe "Rhamnosus" das unspezifische Immunsystem trainiert und ähnlich wie ein Adjuvans bei einer Impfung aktiviert. Die dendritischen Zellen reifen zu kompetenten Makrophagen, die viele körperfremde Partikel fressen - nicht nur Bakterien, sondern auch Viren!

Gegen Corona liegen für LCR 35 noch keine Daten vor. Chinesische
Ärzte empfehlen aber schon jetzt einen Therapie-Mix mit Probiotika [1].

Pädiater der Universität Taipeh in Taiwan behandelten an Rotaviren erkrankte Kinder mit LCR35. Während in der Kontrollgruppe die Erkrankung persistierte, wurde unter LCR35 innerhalb von 3 Tagen die Konzentration der Rotaviren um $86 \%$ abgesenkt. Man kann annehmen, dass der L. casei rhamnosus (LCR35) auch viele andere Viren inaktivieren könnte. Die Studien der nächsten Jahre werden zeigen, wie stark die Schutzwirkung dieses Breitband-Probiotikums bei Kindern ist.

\section{Korrespondenzadresse}

\section{Assoc. Prof. Dr. Christoph Steininger}

Dept. of Medicine I, Medical University of Vienna

Währinger Gürtel 18-20, 1090 Wien,

Österreich

christoph.steininger@meduniwien.ac.at

\section{Literatur}

1. Liang T Handbuch der Zhejiang Universität

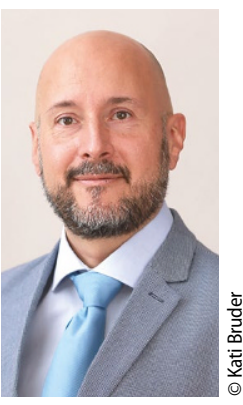

Assoc. Prof. Dr. Christoph Steininger

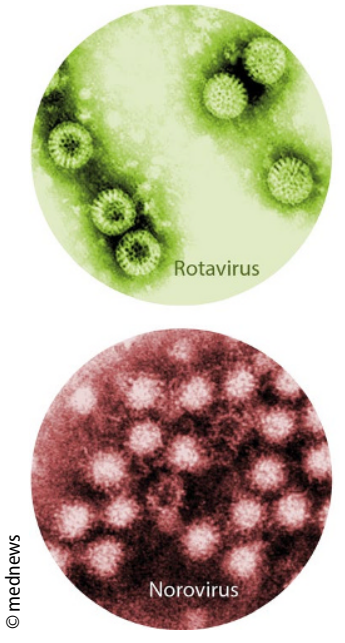

Abb. 14
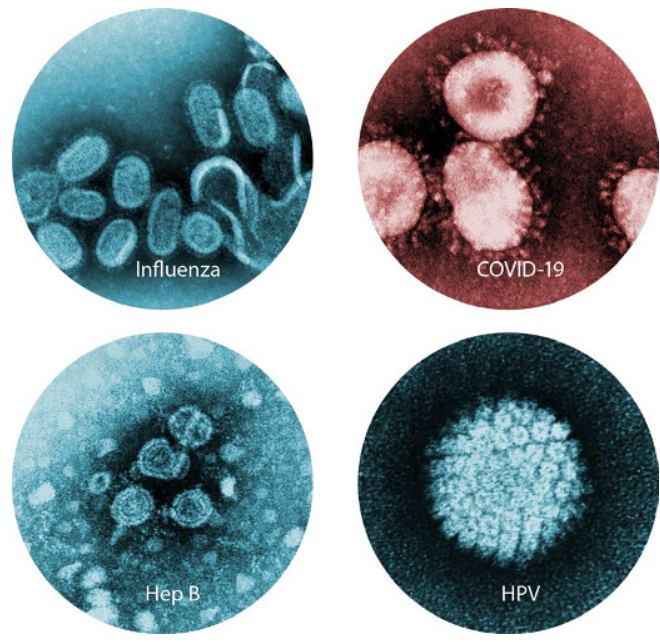

Hinweis des Verlags. Der Verlag bleibt in Hinblick auf geografische Zuordnungen und Gebietsbezeichnungen in veröffentlichten Karten und Institutsadressen neutral.

Paediatr. Paedolog. 2020 · 55:151 https://doi.org/10.1007/s00608-02000791-4

(c) Springer-Verlag GmbH Austria, ein Teil von Springer Nature 2020 